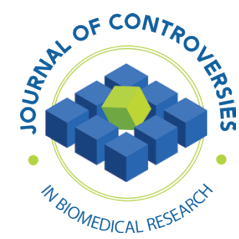

REVIEW ARTICLE

\title{
A Systematic Review of Clinical Trials Testing CETP and PCSK9 Inhibitors: The Cholesterol-Heart Theory-Time for a Requiem?
}

\author{
Mikael Rabaeus ${ }^{1}$, Michel de Lorgeril ${ }^{2}$ \\ ${ }^{1}$ Swiss-mc medical center, Genève, Switzerland; ${ }^{2}$ MDL Etudes et Conseils, Meylan, France
}

\begin{abstract}
There is an ongoing intense controversy around cholesterol lowering using statins, questioning the reality of the benefits and the safety of this treatment. Going even further, this has led to a growing questioning of the robustness of the well-established cholesterol-heart theory, stating that high cholesterol levels ineluctably and strongly increase the risk of coronary artery obstruction and acute myocardial infarction. In the same way, many scientists no longer agree with the theory that high cholesterol increases the risk of ischemic stroke. To test the cholesterol-heart theory, the present systematic review aimed at examining whether the most recent clinical trials testing powerful cholesterol-lowering interventions (such as anti-CETP and anti-PCSK9) report effective reduction of fatal cardiovascular complications and improved survival. Because of high heterogeneity between studies, a meta-analysis was not feasible. The review did show that neither anti-CETP nor anti-PCSK9 treatment can significantly reduce the risk of cardiovascular death, thereby giving credit to the questioning of the cholesterol-heart theory. Our review also shows that the quality of the included trials is generally poor with suspicion of inefficient blinding. This undermines the validity of the reported nonfatal events and thereby increases the importance of comparing fatal endpoints in both groups to test the cholesterol-heart theory.
\end{abstract}

Keywords: anti-CETP medical drugs; anti-PCSK9 medical drugs; cholesterol-heart theory; myocardial infarction; statins

Received: 18 September 2019; Accepted after revision: 20 October 2019; Published: 18 November 2019

Author for correspondence: Mikael Rabaeus, Swiss-mc medical center, 1201 Genève, Switzerland. Email: mikael.rabaeus@bluewin.ch

How to cite: Rabaeus M, de Lorgeril M. A systematic review of clinical trials testing CETP and PCSK9 inhibitors: The cholesterol-heart theory-Time for a requiem? J Controversies Biomed Res. 2019;5(1):4-11.

Doi: https://dx.doi.org/10.36255/jcbmr.2019.35

Copyright: The Author.

License: This open access article is licensed under Creative Commons Attribution 4.0 International (CC BY 4.0). http://creativecommons.org/ licenses/by/4.0

\section{Introduction}

The general perception among many physicians is that public trust in medical science is eroding, leading to unexpected difficulties for everyday medical practices. Loss of public confidence is a consequence of multiple factors: the growing complexity of modern medicine, the perception that experts are often changing assessments of evidence, repeated failures to uphold professional standards, lack of independence of the experts, and finally endless controversies between experts.

The controversy about the cholesterol-lowering statins is of particular interest because millions of people all over the world are taking statins every day with the hope of protecting themselves from cardiovascular disease complications such as myocardial infarction, stroke, and cardiovascular death. The statin treatment is based on the traditional cholesterol-heart theory stating that high cholesterol levels ineluctably result in coronary artery obstruction and acute myocardial infarction, a claim that is strongly challenged at present.

Indeed, as shown in previous reports $(1,2)$, statins are likely not effective for cardiovascular-disease prevention and for prolonging life. One should note that the studies published before 2005/2006 (and the Vioxx tragedy) were probably flawed, and this concerned in particular the efficacy issue $(1,2)$. This is supported by the fact that it is quite common 
to notice serious discrepancies in analyses done by industry-sponsored authors and independent authors (3-8).

In particular, whether statins improve survival in randomized clinical trials (RCTs) is a critical issue as mortality remains the major endpoint to be considered in RCTs testing any cardiovascular medication. The way the commercial RCTs are conducted and the ways the clinical endpoints are recorded, validated, and analyzed are open to major biases $(1,2)$. In the absence of open access to the raw data for independent scientists, it is clear that the mortality endpoint should be the main criterion to test efficacy. It is indeed quite easy to consult death certificates on national registers to know whether at a certain moment of a trial, one patient is still alive or not. In the context of cardiovascular prevention, cardiovascular death should be the major endpoint in the statistical analyses. However, there are clinical circumstances (for instance when there are several diseases in the same patients) where the exact cause of death can be difficult to identify. For this reason, overall mortality data should be analyzed in parallel with cardiovascular mortality to confirm or infirm the cardiovascular mortality endpoint.

It seems evident that in case of a disagreement between experts, the views of the independent authors should be preponderant because the industry-sponsored authors may be influenced in favor of the commercial interests of their sponsors. As industry-sponsored authors are often strongly linked to the academic societies, to the national and international health authorities, and to members of the editorial committees of most influential international journals,their views - obtain the paradoxical support of a majority of scientists and physicians, as if the independent experts were simply a small minority devoted to defending highly controversial opinions.

Once it has been admitted that experts working with the statin industry and those independent of the industry have problems reaching a consensus about the efficiency and/ or toxicity of statins, one of the principal solutions to still discuss the cholesterol-heart theory is to examine the effects of medical substances different from the statins but with a strong effect on cholesterol and lipoproteins. The main question should be, "Having shown demonstrated efficacy in decreasing the so-called bad cholesterol and increasing the good cholesterol, do these substances protect against cardiovascular disease complications?"

If cholesterol-lowering treatments other than statins reduce the risk of cardiovascular complications, it would confirm that the cholesterol-heart theory is correct. However, if these substances fail to reduce the risk, the cholesterol-heart theory should be rejected.

Two families of cholesterol-lowering medications have been tested during the last decade, giving us the opportunity to test whether lowering the so-called "bad" low-density lipoprotein cholesterol (LDL) cholesterol leads to cardiovascular protection. The main aim of the present systematic review is not to examine whether such medications could be prescribed to at-risk patients, but only to test whether the cholesterol-heart theory is still credible.

The two groups of medications are (i) the cholesteryl ester transfer protein (CETP) inhibitors, which we will call anti-CETP in the present study, and (ii) the proprotein convertase subtilisin/kexin type 9 serine protease (PCSK9) inhibitors, which we will call anti-PCSK9 in this study.

The purpose of this study is to examine our present knowledge about the anti-CETP and anti-PCSK9 medical drugs. Are they clinically useful? Do they confirm the statin data? Do they support the cholesterol-heart theory?

\section{Methods}

\section{Search strategy}

We identified all published RCTs testing either an anti-CETP or an anti-PCSK9 against a placebo via a MEDLINE search using the following key words "anti-CETP," "CETP inhibitors," "anti-PCSK9," "PCSK9 inhibitors," "placebo," "cardiovascular disease," "ischemic heart disease," "stroke," "myocardial infarction," and "mortality." The search was updated in March 2019 and retained only double-blinded RCTs designed to examine the effects of an anti-CETP or an anti-PCSK9 medication on cardiovascular outcomes and mortality over a period of at least 12 months. Only RCTs specifically designed to test the effects of anti-CETP or anti-PCSK9 on major clinical outcomes were retained. Clinical studies, whether RCT or not, aimed at principally testing the effects of anti-CETP or anti-PCSK 9 on blood lipids were not retained. Among RCTs reporting clinical endpoints, we only retained those reporting at least a total of 100 events. In addition, we examined the reference lists and related links of retrieved articles that met the inclusion criteria, and also expert reviews and published meta-analyses to detect all studies potentially eligible for inclusion. Cross-sectional, cohort, case-control, and meta-analysis studies were not included. Secondary and/or subgroup analyses studies were excluded because they are performed a posteriori after unblinding, a major cause of bias. We also performed a MEDLINE search to specifically identify double-blinded RCTs testing an anti-CETP or an anti-PCSK9 in patients with specific risk factors such as diabetes. Again, secondary and subgroup analyses studies were excluded. The selection was done by de Logeril and verified by Rabaeus and there was no discrepancy between them. Because of major clinical and biological heterogeneity of the published RCTs, a meta-analysis was not feasible.

\section{Results}

The clinical anti-CETP efficacy data

We retained (Figure 1) four RCTs having appropriately tested anti-CETP drugs (9-12). Main data and results of the four RCTs are presented in Tables 1 and 2. 


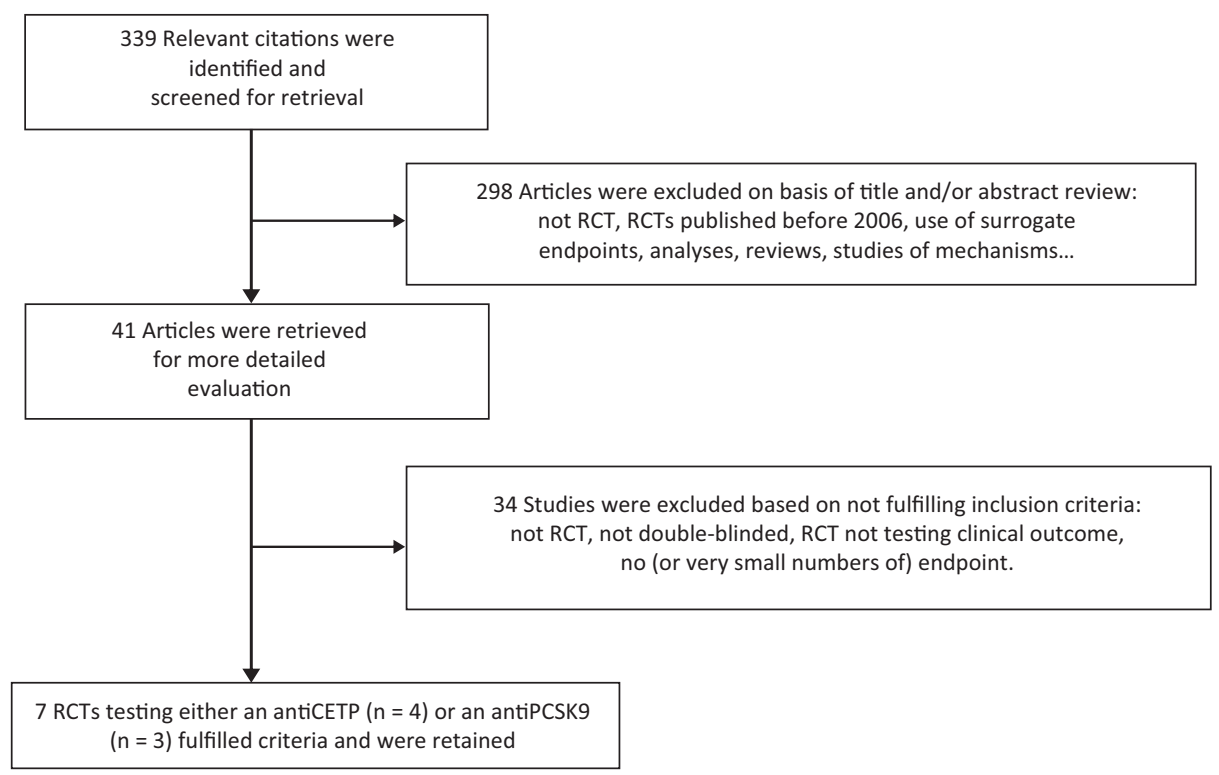

Figure 1. Flow diagram of selection of RCTs testing anti-CETP and anti-PCSK9 drugs.

Table 1. List of the main RCTs testing anti-CETP and discussed in this review.

\begin{tabular}{|l|l|l|l|l|l|l|}
\hline RCT acronym (ref.\#) & \multicolumn{1}{|c|}{ Drug } & Patients & $\begin{array}{c}\text { Follow-up } \\
\text { (months) }\end{array}$ & Samples & $\begin{array}{c}\text { Lipid changes } \\
\text { LDL and HDL }\end{array}$ \\
$\begin{array}{l}\text { outcome Hazard } \\
\text { ratio (95\% CI) }\end{array}$ \\
\hline Illuminate(9) & Torcetrapib & Stable CHD & 18 & 15,067 & $-28 \%+70 \%$ & $1.25(1.09-1.44)$ \\
\hline DAL Outcomes(10) & Dalcetrapib & ACS & 31 & 15,871 & $0+27 \%$ & $1.04(0.93-1.16)$ \\
\hline Accelerate(11) & Evacetrapib & ACS and stable CHD & 26 & 12,092 & $-37 \%+132 \%$ & $1.01(0.91-1.11)$ \\
\hline Reveal(12) & Anacetrapib & Stable CHD & 49 & 30,449 & $-41 \%+104 \%$ & $0.91(0.85-0.97)$ \\
\hline
\end{tabular}

Note: CHD, coronary heart disease; RCT, randomized clinical trial; ACS, acute coronary syndrome; CI, confidence interval; LDL, low-density lipoprotein cholesterol; HDL, high-density lipoprotein cholesterol.

The results of the Illuminate trial (9) were published in 2007 at a time when, after the Vioxx incident, investigators and sponsors were extremely prudent about respecting the ethics and methods of clinical research because they knew that, in case of nonrespect of regulations, they risk a prison sentence or at least paying a large fine (13-18). As described by the authors, Illuminate was a randomized, double-blind study involving 15,067 patients at high cardiovascular risk. The patients received torcetrapib (an anti-CETP) plus atorvastatin or atorvastatin plus placebo. The trial was terminated prematurely because of an increased risk of death (Table 2) and cardiac events in patients receiving torcetrapib. These unexpected complications occurred despite the fact that in patients who received torcetrapib, there was an increase of $70 \%$ in high-density lipoprotein cholesterol (HDL) and a decrease of $28 \%$ in LDL, as compared with patients receiving placebo (Table 1). There was also an increased risk of nonfatal cardiovascular events (HR, 1.25; $\mathrm{P}=0.001)$ and death from noncardiovascular causes (HR, 1.58; $\mathrm{P}=0.006)$. In particular, there were more deaths from cancer (24 vs. 14) and from infection ( 9 vs. 0 ) in the anti-CETP group compared with the placebo group. The authors explained these catastrophic results by an increase of $5.4 \mathrm{mmHg}$ in systolic blood pressure and a small decrease in serum potassium, a conclusion which seems somewhat curious.

The dal-OUTCOMES trial (10) was published in 2012. It was a randomized, double-blind study involving 15,871 patients who had had a recent acute coronary syndrome (ACS) to receive either the anti-CETP dalcetrapib or matching placebo (Table 1). Over the course of the trial, HDL increased by $27 \%$ on an average in the dalcetrapib group. Dalcetrapib had a nonsignificant effect on LDL cholesterol 
Table 2. Summary of mortality data in the four anti-CETP trials

\begin{tabular}{|l|r|r|}
\hline Cardiovascular mortality & & Placebo \\
\hline Illuminate & 35 & 49 \\
\hline DAL outcomes & 135 & 122 \\
\hline Accelerate & 166 & 143 \\
\hline Reveal & 563 & 517 \\
\hline Total & 899 & $831(-7 \%)$ \\
\hline Overall mortality & & \\
\hline Illuminate & 55 & 89 \\
\hline DAL outcome & 222 & 226 \\
\hline Accelerate & 276 & 1126 \\
\hline Reveal & 1157 & $1672(-2 \%)$ \\
\hline Total & 1710 & \\
\hline
\end{tabular}

Table 3. Summary of mortality data in the three anti-PCSK 9 trials

\begin{tabular}{|l|r|r|}
\hline Cardiovascular mortality & Placebo & anti-PCSK9 \\
\hline SPIRE & 64 & 65 \\
\hline FOURIER & 240 & 251 \\
\hline ODYSSEY & 271 & 240 \\
\hline Total & 575 & $556(-3 \%)$ \\
\hline Overall mortality & & \\
\hline SPIRE & 117 & 120 \\
\hline FOURIER & 426 & 444 \\
\hline ODYSSEY & 392 & 334 \\
\hline Total & 935 & $898(-4 \%)$ \\
\hline
\end{tabular}

levels. Patients were followed up for a median of 31 months. At a prespecified interim analysis that included $71 \%$ of the projected total number required to test the primary hypothesis, the data and safety monitoring board recommended termination of the trial for futility. Despite significant increase in the supposed protective HDL, dalcetrapib did not alter the risk of the primary end point $(\mathrm{HR}, 1.04 ; \mathrm{P}=0.52)$ and did not have a significant effect on any component of the primary end point (including cardiovascular mortality) or total mortality (Table 2). There was no between-group difference in systolic blood pressure and potassium (10). There was no convincing explanation for the failure of the trial.

The Accelerate trial was published in 2017 (11). It is a randomized, double-blind study involving 12,092 patients who had at least one of the following conditions: an ACS, cerebrovascular atherosclerotic disease, peripheral vascular arterial disease, or diabetes mellitus with stable CHD. Patients were randomly assigned to receive either the anti-CETP evacetrapib or matching placebo (Table 1). After 3 months, a $37 \%$ decrease of LDL and a $133 \%$ increase of HDL were observed with evacetrapib compared with placebo. After 26 months of follow-up, 12.9\% of the patients in the evacetrapib group and $12.8 \%$ of those in the placebo group (HR, 1.01; $\mathrm{P}=0.91$ ) had a primary endpoint event. There was no significant between-group difference for cardiovascular and overall mortality (Table 2). Therefore, the data and safety monitoring board recommended that the trial be terminated early because of a lack of efficacy. It was the third anti-CETP trial to be prematurely terminated indicating both a lack of efficacy and a lack of permanent blinding. Authors did not propose a convincing explanation for the lack of efficacy despite striking effects on both LDL (decrease) and HDL (increase).

The HPS3/TIMI55-REVEAL Collaborative Group trial (12) was published in 2017. It was a randomized, double-blind study involving 30,449 patients with stable coronary heart disease (CHD). The patients were assigned to receive either the anti-CETP anacetrapib or matching placebo. At the trial midpoint, the mean level of HDL in the anacetrapib group was increased by $104 \%$ and the mean level of LDL was decreased by $41 \%$ (direct method) compared with the placebo group (Table 1). After a mean follow-up of 49 months, authors claimed that the primary outcome occurred in significantly fewer patients in the anacetrapib group than in the placebo group ( $10.8 \%$ vs. $11.8 \%$; HR, $0.91 ; \mathrm{P}=0.004)$. There were no significant between-group differences in cardiovascular and overall mortality (Table 2). Because of these dubious results, Merck decided not to file for approval of anacetrapib (19).

Thus, after the sad failure of the three previous anti-CETP trials, the anti-CETP drug class finally died as Merck abandoned anacetrapib. "We are deeply grateful to the researchers and patients who participated in the anacetrapib clinical development program, and in particular the REVEAL outcomes study. Unfortunately, after comprehensive evaluation, we have concluded that the clinical profile for anacetrapib does not support regulatory filings," commented Roger M. Perlmutter, president of Merck (20). Actually, when adding the mortality data in the four anti-CETP trials (Table 2), it appears that anti-CETP substances may decrease cardiovascular mortality by $7 \%$ and overall mortality by $2 \%$. However, for epidemiologists and in terms of public health, these data do not support the use of anti-CETP medication. Contrary to some experts who still claim that anti-CETP may reduce the risk of nonfatal complications, the sponsors and independent scientists know that, in the context of commercial RCTs, only significant effect on the risk of death should be considered. 


\section{The clinical anti-PCSK9 efficacy data}

We retained (Figure 1) three RCTs having appropriately tested anti-CPSK 9 medications $(21,22,23)$.

The results of the SPIRE Cardiovascular Outcome trial were published in 2017 (21). It is a large randomized, double-blind trial combining two parallel trials with different entry criteria for LDL. Investigators randomly assigned 27,438 patients in the combined trials to subcutaneously receive bococizumab (a monoclonal antibody that inhibits PCSK9) or placebo every 2 weeks. The primary end point was nonfatal myocardial infarction, nonfatal stroke, hospitalization for unstable angina requiring urgent revascularization, or cardiovascular death. The trials were stopped early after the sponsor elected to discontinue bococizumab owing in part to the development of high rates of antidrug antibodies in other studies. At 14 weeks, patients in the combined trials had a mean LDL change of $-56.0 \%$ in the bococizumab group and a between-group difference of $-59 \%$ compared with the placebo group $(\mathrm{P}<0.001)$ while between-group difference in HDL was $6 \%$. After a median follow-up of 10 months, major cardiovascular events included in the primary hypothesis occurred in 352 patients in the bococizumab group and 397 patients in the placebo group (HR, 0.88; $\mathrm{P}=0.08$ ). More importantly, there was no between-group difference in cardiovascular mortality ( 65 deaths with bococizumab vs. 64 with placebo) and mortality from any cause (120 deaths with bococizumab vs. 117 with placebo). It is noteworthy that the numbers of serious adverse events were not different in the two groups (1995 with bococizumab and 1999 with placebo). Authors claimed that the SPIRE trials "were terminated early by the sponsor owing to the development of antidrug antibodies in other studies in the program." This premature termination was not justified by the advent of major side effects or by a neutralizing effect of antidrug antibodies with limitation of the cholesterol-lowering effect. In fact, along with a greater incidence of injection-site reactions with bococizumab compared with other drugs in the class, it is likely that, in view of the weak clinical cardiovascular efficiency observed in SPIRE, Pfizer anticipated a poor commercial outcome of that anti-PCSK9 drug (22).

The results of the FOURIER trial were published in 2017 (23). It was a randomized, double-blind study involving 27,564 patients with stable CHD. Patients were randomly assigned to receive either evolocumab (a monoclonal antibody that inhibits PCSK9) or matching placebo as subcutaneous injections. The primary efficacy end point was the composite of cardiovascular death, myocardial infarction, stroke, hospitalization for unstable angina, or coronary revascularization. All along the follow-up, the difference in LDL between groups varied from $54 \%$ to $61 \%$ (24). Relative to placebo, investigators claimed that after a mean follow-up of 26 months, evolocumab reduced the risk of the primary end point: $9.8 \%$ of the patients with evolocumab versus $11.3 \%$ with the placebo had an endpoint: HR, 0.85; P $<0.001$.
However, there was no significant between-group difference in cardiovascular mortality (251 deaths with evolocumab vs. 240 with placebo) and mortality from any cause (444 deaths with evolocumab vs. 426 with placebo). In addition, death or hospitalization for worsening heart failure (a strong clinical endpoint) was not different in the two groups: 402 events with evolocumab vs. 408 with placebo. Thus, the main difference between the two groups was for coronary revascularization (759 vs. $965 ; \mathrm{P}<0.001)$ and nonfatal myocardial infarction (468 vs. $639 ; \mathrm{P}<0.001$ ), two endpoints considered weak in the context of commercial RCTs $(1,2)$. For instance, the increased use of highly sensitive cardiac troponin assays, in association with the lack of strict blinding, results in possible confusion in the diagnosis of acute myocardial infarction versus unstable angina $(1,2)$. In the FOURIER trial, there was a between-group difference for myocardial infarction but not for unstable angina (236 events with evolocumab vs. 239 with placebo). This is somewhat surprising as the pathophysiology of both is very similar. These clinical discrepancies in the context of poor blinding do not help trusting the data regarding nonfatal myocardial infarction and therefore the primary endpoint. Only a full access to the raw database and verification of each endpoint could convince independent scientists that evolocumab is better than placebo. Overall, the results of the FOURIER trial were quite disappointing and very similar to those of the REVEAL trial testing the anti-CETP anacetrapib and discussed earlier (12).

The results of the ODYSSEY OUTCOMES trial were published in 2018 (24). The ODYSSEY trial was a randomized, double-blind study involving 18,924 patients who had an ACS 1-12 months earlier. Patients were randomly assigned to receive alirocumab (a monoclonal antibody that inhibits PCSK9) subcutaneously at a dose of $75 \mathrm{mg}$ or a matching placebo every 2 weeks. The dose of alirocumab was adjusted to target LDL levels of $0.6-1.3 \mathrm{mmol} / \mathrm{L}$. The primary end point was a composite of death from CHD, nonfatal myocardial infarction, fatal or nonfatal ischemic stroke, or unstable angina requiring hospitalization. Interim analyses were planned during the trial and adjustments of doses of alirocumab were required during the trial after repeated measurements of LDL. It therefore seems justified to be skeptical about the real respect of double-blinding during the whole trial. Only careful analyses of the raw dataset would allow establishing that biases were not introduced because of lack of blinding. In the alirocumab group, the LDL levels at 4 and 12 months were an average of $63 \%$ and $61 \%$ lower than the respective levels in the placebo group. The authors report that after a median follow-up of 34 months, the composite primary endpoint occurred in $9.5 \%$ of the patients in the alirocumab group and in $11.1 \%$ in the placebo group (HR, 0.85; $\mathrm{P}<0.001$ ). However, mortality from CHD was not significantly different between groups: 205 deaths with alirocumab versus 222 with placebo. Also, hospitalization for worsening heart failure was similar in both groups: 176 events 
with alirocumab versus 179 with placebo. But then, authors claim that a total of 334 patients in the alirocumab group and 392 patients in the placebo group died: HR, 0.85; 95\% CI, 0.73-0.98 (Table 3). This is an important issue because overall mortality was not included in the primary hypothesis. The lack of significant effect on total cardiovascular mortality (240 deaths with alirocumab vs. 271 with placebo) in contrast to a reduction of overall mortality should raise the suspicion of an effect of chance, all the more when considering the suspicion of bias in the recording and the analyses of the data. In the context of a commercial trial, these discrepancies and probable lack of blinding do raise suspicion concerning the data regarding the primary endpoint. Once again, only a full access to the raw database and verification of each endpoint could convince independent scientists that alirocumab is better than placebo. Overall, the results of the ODYSSEY trial were quite disappointing and very similar to those of the REVEAL trial testing the anti-CETP anacetrapib and discussed above (12).

\section{Summary of the data}

We have analyzed seven RCTs testing the effects of the cholesterol-lowering anti-CETP and anti-PCSK9 on the risk of cardiovascular complications. These RCTs essentially involved patients in secondary prevention (stable CHD or after a recent ACS), a clinical situation expected to be the most appropriate to test an effect on major complications, in particular cardiovascular death.

As shown in Tables 2 and 3, anti-CETP or anti-PCSK9 medication resulted in a non-significant $2 \%-4 \%$ reduction of overall mortality and 3\%-7\% reduction of cardiovascular mortality, the two main outcomes to be considered in the first place when examining the results of commercial trials, as discussed in the "Introduction" section.

With the exception of the DAL-Outcome trial, the cholesterol-lowering effect was impressive in these trials but this did not result in better survival. In other words, intense cholesterol-lowering using drugs different from statins failed to show significant protection from CHD and better survival. It is noteworthy that striking increases in HDL also did not result in significant benefit. As the idea that HDL (the so-called "good" cholesterol) protects against cardiovascular complication is a major element of the cholesterol-heart theory, this failure of the anti-CETP medication is a strong argument to question the theory.

Overall, the anti-CETP and anti-PCSK9 trial data do not confirm the supposed protective effect of cholesterol-lowering, as has been claimed with statins (3-5), and thereby confirm the skepticism of independent scientists $(1,2,6)$.

It could be said that despite the lack of effect on cardiovascular mortality, treatment with anti-CETP or anti-PCSK9 resulted in less cardiovascular morbidity or less nonfatal complications. In fact, there were many discrepancies (discussed in the "Results" section for each trial) in the results regarding nonfatal complications as there were many methodological limitations in each trial, in particular the lack of consistent double-blinding, for instance, when interim analyses are planned and dose adjustment is required. Only a full access to the raw database and verification of each endpoint in each trial could convince independent scientists that anti-CETP and anti-PCSK 9 medication are better than placebo.

It is noteworthy that most sponsors themselves were convinced that these cholesterol-lowering drugs had a poor commercial future and therefore did not apply for regulatory filings to place these medications on the market. All the anti-CETP and one anti-PCSK9 (bococizumab) have been abandoned by the companies which own the patents.

The two exceptions are Sanofi and Amgen (about their anti-PCSK9) but the marketing authorizations they have obtained for evolocumab and alirocumab were for the treatment of hypercholesterolemia and not for the prevention of CHD complications. For instance, the French Health Authorities said for evolocumab that "no benefit was observed regarding overall and cardiovascular mortality and quality of life" (25). Regarding alirocumab, the same French Health Authorities, surprisingly stated that "in high-risk coronary patients with hypercholesterolemia, it could be used only in association with other cholesterol-lowering drugs" (26).

To save the cholesterol-heart theory, scientists have curiously proposed new definitions of "LDL cholesterol" (27). They say that "LDL cholesterol is an important contributor to the risk of coronary heart disease, and its measurement is central to evaluating the effects of lipid-modifying therapies. Several LDL assays exist but their methodologies differ, leading to between-assay heterogeneity in values of LDL." Given the failure of LDL reduction to reduce the risk of death in the seven RCTs analyzed in this review, and also in most statin trials when analyzed by independent investigators $(1,2$, 6), it is unlikely that "between-assay heterogeneity in values of $L D L$ " could explain the data.

Other experts have claimed that the failure of anti-CETP medication to reduce the risk of death, despite significant effect on the lipid profile (increasing the "good" cholesterol, decreasing the "bad"), could be explained by "unanticipated off-target effects that increased risk of death" for certain anti-CETP substances or by their "accumulation in adipose tissue" (anacetrapib) (28).

In fact, for most experts defending the cholesterol-heart theory, it is simply impossible to admit that the theory is dead, as this would mean that an inefficient and potentially toxic medication has been prescribed to millions of patients over the world during the last decades.

\section{Limitations of the study}

There are some limitations in the present study. The main limitation is that we do not have free access to the raw data 
of the RCTs we are discussing. The lack of transparency of commercial RCTs - that is, the impossibility of verifying the way the clinical data are collected, how the datasets are constituted, cleaned, and digitized, and how the statistical analyses are conducted - definitely limits our ability to evaluate the accuracy of the published data.

In fact, as the seven commercial RCTs we have included in our analysis were more or less negative, it is unlikely that investigators did not do their best to prevent any bias that could be contra to the tested medication. In some RCTs, however, it could have been useful to get access to the raw data to explain unexpected data, for instance the decreased overall mortality in ODYSSEY (23) or the decrease in nonfatal myocardial infarction in REVEAL (12).

Another limitation is the lack of meta-analysis. As discussed in the "Introduction" section, the anti-CETP medications had quite a different effect on the lipid profiles with decrease in LDL varying from $41 \%$ to nothing and increase in HDL varying from $27 \%$ to $132 \%$. In fact, the present study aimed at examining whether specific changes in lipid profiles with nonstatin medication may result in clinical results that confirm the supposed benefits of cholesterol-lowering. In that context, meta-analysis was not the appropriate method. In contrast, as shown in Table 2, the simple addition of the numbers of deaths in each RCT clearly shows that CETP inhibition does not result in any benefit.

The same reasoning applies for PCSK9 inhibition. The monoclonal antibodies tested in the three RCTs had different biological effects, as discussed in the "Result" section, and again the simple addition of the numbers of deaths (Table 3) provides highly significant clinical information.

Finally, one possible limitation is that anti-CETP and anti-PCSK9 medications were generally tested in patients receiving high statin doses or in patients with already low LDL because of statin use. Despite the fact that CETP and PCSK9 inhibition resulted in highly significant additional reduction of LDL (in terms of percentage) - and significant increase of HDL with anti-CETP inhibition - it is not clear whether these medications could have been useful in the absence of statin.

This possibility is in contradiction with the conventional cholesterol-heart theory which claims that "the lower the better." At any baseline LDL, an additional LDL decrease should result in additional clinical benefits. Investigators and sponsors were aware of the fact that clinical benefits might be more difficult to observe if baseline LDL was already low. To offset this difficulty, sample sizes in the seven RCTs were huge compared with the first statin RCTs. For instance, in the $4 \mathrm{~S}$ study testing simvastatin, only 4444 patients were randomized while a highly significant reduction of mortality was observed (29). In the seven RCT we reviewed, sponsors and investigators also took the precaution of recruiting high-risk patients in secondary prevention (as in $4 \mathrm{~S}$ ) with an expected high rate of complications over a quite short follow-up. Finally, it is quite noteworthy that none of the sponsors did claim that the tested anti-CETP and anti-PCSK9 could have been more efficient in the absence of statins.

\section{Conclusion}

The aim of this study was to examine the effects of cholesterol-lowering by agents other than statins on clinical events. The review we conducted shows that despite a very significant effect on cholesterol levels, the CETP and PCSK9 inhibitors have not been shown to diminish the frequency of clinical events in high-risk patients, especially not the important ones represented by total and cardiovascular deaths. The only way to confirm or infirm this would be to have access to raw data, especially in view of the suspicion of insufficient blinding. Another consequence of these findings is that they speak strongly against the cholesterol-heart theory, confirming the doubts that have already been raised by a large group of scientists all over the world. As this theory leads to millions of people taking statin drugs, it appears highly necessary that access to raw data of all statin trials be allowed so as to reappreciate them. This is an important aspect considering the very strong conflicts of interest that the majority of scientists present, all the more concerning as many of these scientists exercise official activities in Association boards and guidelines committees and in medical journals. Therefore, we continue to maintain that the cholesterol-heart theory should be seriously challenged.

\section{Conflict of Interest}

None of the authors have any financial links with the pharmaceutical industry. Michel de Lorgeril discloses receiving an honorarium from Barilla Inc.

\section{References}

1. de Lorgeril M, Rabaeus M. Beyond confusion and controversy, can we evaluate the real efficacy and safety of cholesterol-lowering with statins? J Controversies Biomed Res. 2015;1:67-92. http://dx.doi.org/10.15586/jcbmr.2015.11

2. Rabaeus M, Nguyen PV, de Lorgeril M. Recent flaws in evidence-based medicine: Statin effects in primary prevention and consequences of suspending the treatment. J Controversies Biomed Res. 2017;3:1-10. http://dx.doi.org/10.15586/ jcbmr.2017.18

3. Collins R, Reith C, Emberson J, Armitage J, Baigent C, Blackwell $\mathrm{L}$, et al. Interpretation of the evidence for the efficacy and safety of statin therapy. J Lancet. 2016;388:2532-61. http://dx. doi.org/10.1016/S0140-6736(16)31357-5

4. Silverman MG, Ference BA, Im K, Wiviott SD, Giugliano RP, Grundy SM, et al. Association between lowering LDL-C and cardiovascular risk reduction among different therapeutic interventions: A systematic review and meta-analysis. J Am Med Assoc. 2016;316:1289-97. http://dx.doi.org/10.1001/ jama.2016.13985

5. Ference BA, Ginsberg HN, Graham I, Ray KK, Packard CJ, Bruckert E, et al. Low-density lipoproteins cause atherosclerotic 
cardiovascular disease. 1. Evidence from genetic, epidemiologic, and clinical studies. A consensus statement from the European Atherosclerosis Society Consensus Panel. Eur Heart J. 2017;38:2459-72. http://dx.doi.org/10.1093/eurheartj/ehx144

6. Ravnskov U, de Lorgeril M, Diamond DM, Hama R, Hamazaki T, Hammarskjöld B, et al. LDL-C does not cause cardiovascular disease: A comprehensive review of the current literature. Expert Rev Clin Pharmacol. 2018;11:959-70. http://dx.doi.org $/ 10.1080 / 17512433.2018 .1519391$

7. Cholesterol Treatment Trialists' Collaboration. Efficacy and safety of statin therapy in older people: A meta-analysis of individual participant data from 28 randomised controlled trials. Lancet. 2019;39:407-15. http://dx.doi.org/10.1016/ S0140-6736(18)31942-1

8. Ravnskov U, Diamond DM, Hama R, Hamazaki T, Hammarskjöld B, Hynes N, et al. Lack of an association or an inverse association between low-density-lipoprotein cholesterol and mortality in the elderly: A systematic review. BMJ Open. 2016;6:e10401. [cited 2005 XXX XX]. Available from: http:// dx.doi.org/10.1136/bmjopen-2015-010401

9. Barter PJ, Caulfield M, Eriksson M, Grundy SM, Kastelein JJ, Komajda M, et al. Effects of torcetrapib in patients at high risk for coronary events. N Engl J Med. 2007;357:2109-22. http:// dx.doi.org/10.1056/nejmoa0706628

10. Schwartz GG, Olsson AG, Abt M, Ballantyne CM, Barter PJ, Brumm J, et al. Effects of dalcetrapib in patients with a recent acute coronary syndrome. N Engl J Med. 2012;367:2089-99. http://dx.doi.org/10.1056/NEJMoa1206797

11. Lincoff AM, Nicholls SJ, Riesmeyer JS, Barter PJ, Brewer HB, Fox KAA, et al. Evacetrapib and cardiovascular outcomes in high risk vascular disease. N Engl J Med. 2017;376:1933-42. http://dx.doi.org/10.1056/NEJMoa1609581

12. HPS TIMI REVEAL Collaborative Group. Effects of anacetrapib in patients with atherosclerotic vascular disease. N Engl J Med. 2017;377:1217-27. http://dx.doi.org/10.1056/NEJMoa1706444

13. Karha J, Topol EJ. The sad story of Vioxx, and what we should learn from it. Cleve Clin J Med. 2004, 71:933-4, 936, 938-9. http://dx.doi.org/10.3949/ccjm.71.12.933

14. Alpert JS. The Vioxx debacle. Am J Med. 2005;118:203-4. http://dx.doi.org/10.1016/j.amjmed.2005.01.020

15. Ioannidis JP. Why most published research findings are false. PLoS Med. 2005;2:e124. http://dx.doi.org/10.1371/journal. pmed.0020124

16. New Clinical Trial Regulation. [cited 2015]. Available from: http://ec.europa.eu/health/files/eudralex/vol-1/dir_2005_28/ dir_2005_28_en.pdf

17. Commission Directive 2005/28/EC of 8 April 2005 laying down principles and detailed guidelines for good clinical practice as regards investigational medicinal products for human use, as well as the requirements for authorization of the manufacturing or importation of such products. Official Journal of the European Communities L91:13-9. Available from: http://ec.europa. eu/enterprise/pharmaceuticals/eudralex/vol-1/dir_2005_28/ dir_2005_28_en.pdf

18. Bollapragada SS, Norrie JD, Norman JE. Review of new regulations for the conduct of clinical trials of investigational medicinal products. BJOG. 2007;114:917-21.

19. Merck abandons Anacetrapib, 2017. [cited 2017]. Available from: http://www.pmlive.com/pharma_news/cetp_inhibitor_ class_finally_dies_as_merck_abandons_anacetrapib_1208239

20. Merck abandons Anacetrapib, 2017. [cited 2017]. Available from: https://investors.merck.com/news/press-release-details/2017/ Merck-Provides-Update-on-Anacetrapib-DevelopmentProgram/default.aspx

21. Ridker PM, Revkin J, Amarenco P, Brunell R, Curto M, Civeira F, et al. Cardiovascular efficacy and safety of Bococizumab in high-risk patients. N Engl J Med. 2017;376:1527-39. http://dx. doi.org/10.1056/NEJMoa1701488

22. Pfizer. Pfizer discontinues global development of bococizumab, its investigational PCSK9 inhibitor. [cited 2016 Nov 1]. Available from: http://www.pfizer.com/news/press-release/ press-releasedetail/pfizer_discontinues_global_development_ of_bococizumab_its_investigational_pcsk9_inhibitor

23. Schwartz GG, Steg PG, Szarek M, Bhatt DL, Bittner VA, Diaz $\mathrm{R}$, et al. Alirocumab and cardiovascular outcomes after acute coronary syndrome. N Engl J Med. 2018;379:2097-107. http:// dx.doi.org/10.1056/NEJMoa1801174

24. Sabatine MS, Giugliano RP, Keech AC, Honarpour N, Wiviott SD, Murphy SA, et al. Evolocumab and clinical outcomes in patients with cardiovascular disease. N Engl J Med. 2017;376:1713-22. http://dx.doi.org/10.1056/ NEJMoa1615664

25. [cited 2018 Nov 28]. Available from: https://www.has-sante.fr/ jcms/c_2869374/fr/repatha-evolocumab-anti-pcsk9

26. [cited 2019 Aug 1]. Available from: https://www.has-sante.fr/ jcms/p_3081881/fr/praluent

27. Holmes MV, Ala-Korpela M. What is "LDL cholesterol"? Nat Rev Cardiol. 2019;16:197-8. http://dx.doi.org/10.1038/ s41569-019-0157-6

28. Armitage J, Holmes MV, Preiss D. Cholesteryl ester transfer protein inhibition for preventing cardiovascular events: JACC review topic of the week. J Am Coll Cardiol. 2019;73:477-87. http://dx.doi.org/10.1016/j.jacc.2018.10.072

29. Scandinavian Simvastatin Survival Study Group. Randomised trial of cholesterol lowering in 4444 patients with coronary heart disease: The Scandinavian Simvastatin Survival Study (4S). Lancet. 1994;344:1383-9. http://dx.doi.org/10.1016/ S0140-6736(94)90566-5 